\title{
Lateral Pharyngeal Space
}

National Cancer Institute

\section{Source}

National Cancer Institute. Lateral Pharyngeal Space. NCI Thesaurus. Code C162818.

A cone-shaped lateral neck space with its base on the base of the skull and its apex at the hyoid bone. 